\title{
ORIGINS AND EVOLUTION OF LIFE
}

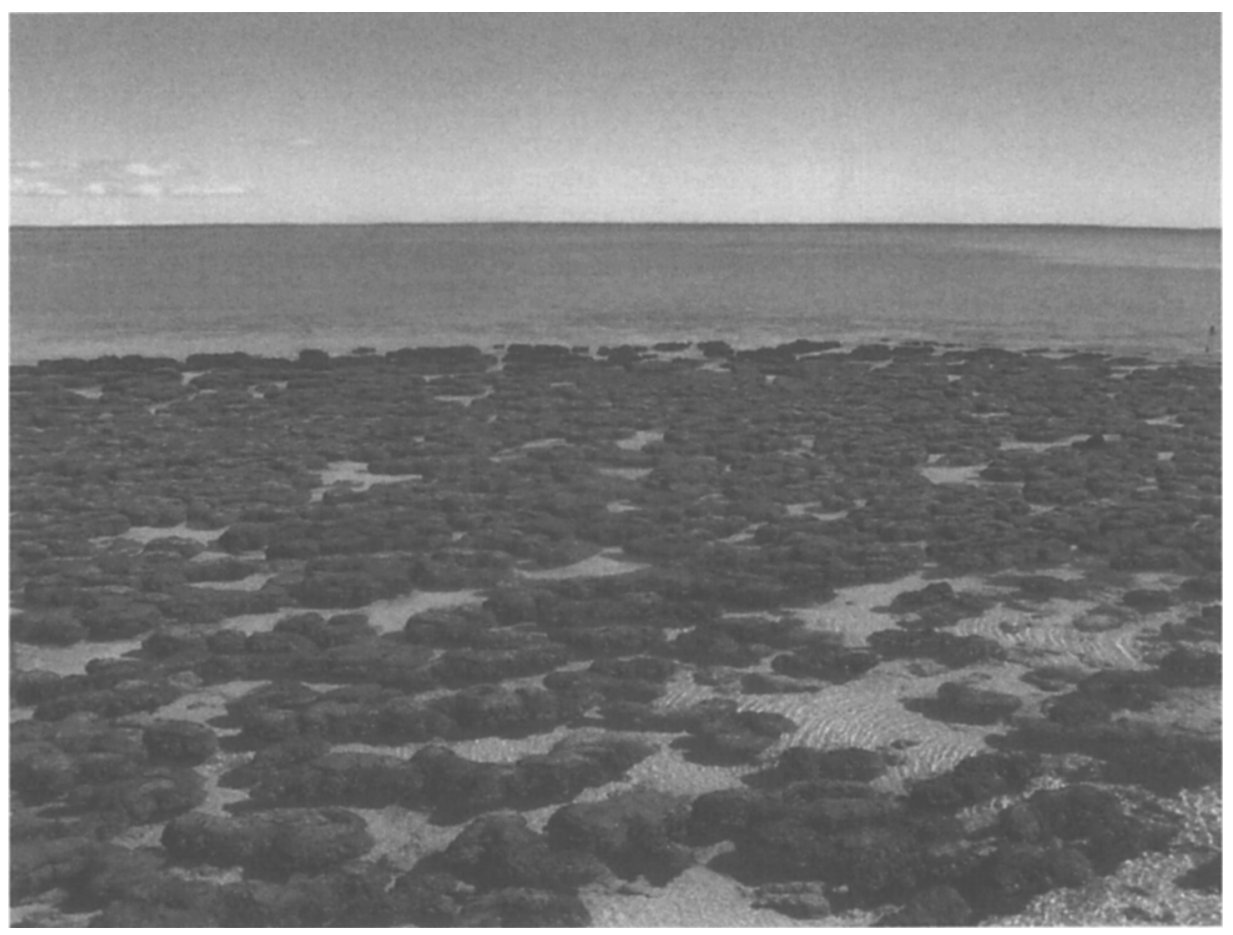

Stromatolites: Shark Bay, Western Australia (photo: Ray Norris) 


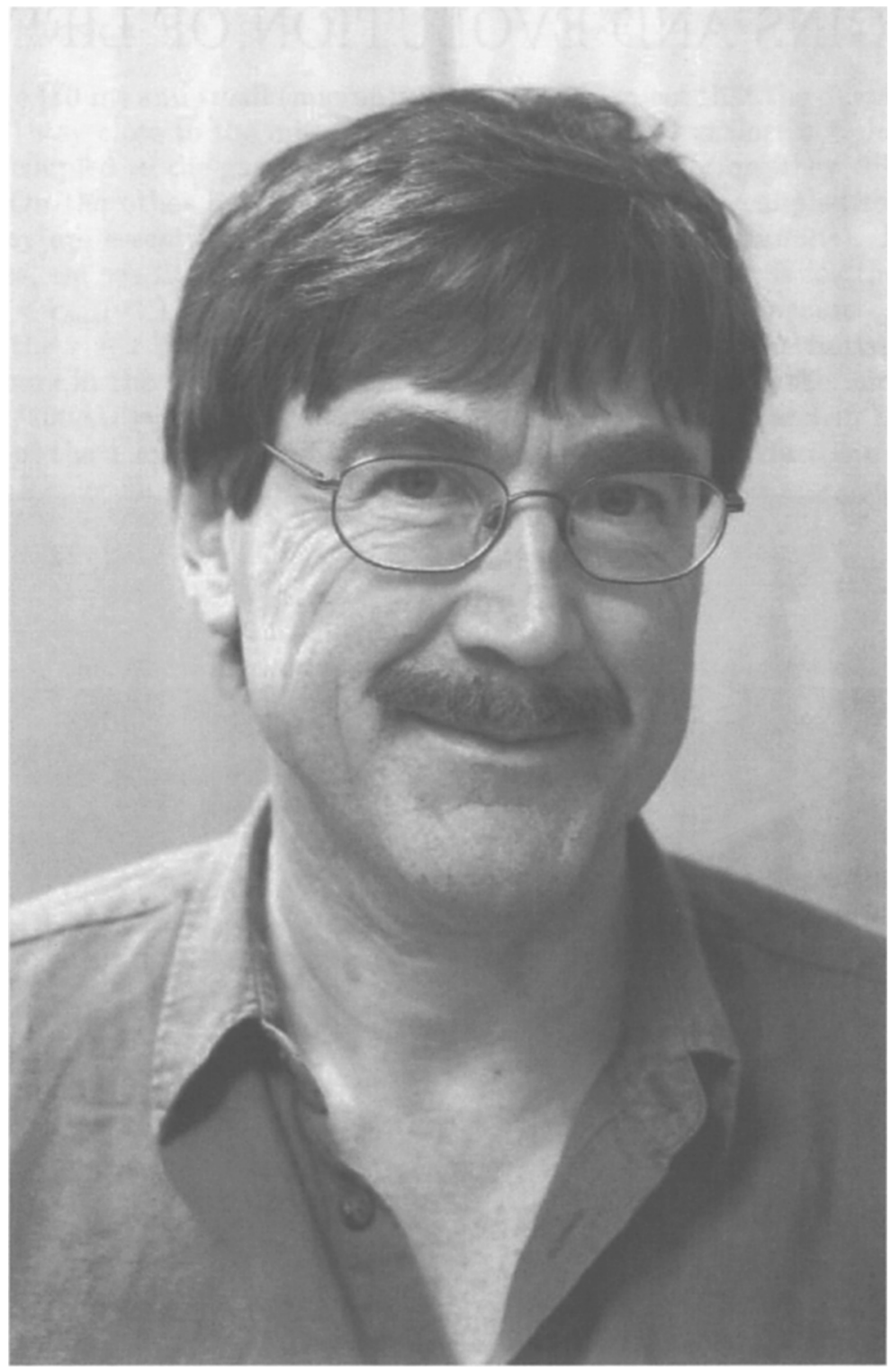

Paul Davies (photo: Seth Shostak) 


\title{
Quantum Mechanics and the Origin of Life
}

\author{
Paul Davies \\ Australian Centre for Astrobiology, Macquarie University, NSW 2109 \\ Australia
}

\begin{abstract}
The race to build a quantum computer has led to a radical re-evaluation of the concept of information. In this paper I conjecture that life, defined as an information processing and replicating system, may be exploiting the considerable efficiency advantages offered by quantum computation, and that quantum information processing may dramatically shorten the odds for life originating from a random chemical soup. The plausibility of this conjecture rests, however, on life somehow circumventing the decoherence effects of the environment. I offer some speculations on ways in which this might happen.
\end{abstract}

It is often remarked that the raw materials for life are widespread in the universe, so therefore life should be widespread too. To be sure, the chemical elements needed by life, C, H, O, N, P and S, are among the most common, while the basic building blocks of life, such as amino acids, form easily and are found in meteorites and probably exist in comets and interstellar clouds too. However, to claim that life is therefore common is to commit the elementary logical fallacy of confusing a necessary with a sufficient condition. The basic building blocks are necessary for life, but they are far from sufficient. One might as well say that because silicon is a common element so we can expect laptop computers to be found throughout the universe.

Nevertheless, the belief that the universe is inherently bio-friendly is currently popular among scientists (although that was not the case thirty years ago). It is a point of view eloquently championed by Christian de Duve, who regards life as 'a cosmic imperative' almost bound to arise wherever physical conditions are similar to those on Earth (de Duve 1995). Robert Shapiro (1986) has called this position biological determinism - the assumption that life is 'written into' the laws of physics, so that given earthlike conditions it will emerge sooner or later as a more or less automatic consequence of those life-encouraging laws.

It is clear, however, that the laws of classical physics (at least as understood so far) are not cunningly rigged in favour of life. There is nothing obvious in those laws to fast-track matter to life against the odds. Nor is this a surprise. The laws of physics are by their very nature simple, mathematical and general, whereas life is a complex, non-mathematical and exceedingly special state of matter. We do not expect the general laws of physics (which apply to everything) to favour, or contain, specific complex states of matter. Indeed, since the time of Newton, there has been a conceptual division between laws and states. To expect life to be 'written into' the laws of physics therefore conflicts with the very basis of physical science as it has been formulated in the last three hundred 
years. It follows that the philosophy of biological determinism contains a hidden assumption of a cosmic imperative or life principle that lies beyond the domain of classical physics.

But before dismissing biological determinism, it is worth enquiring where such a principle might lurk. Traditionally it has been sought in organic chemistry. The success of the Miller-Urey experiment (Miller 1953) in synthesizing amino acids encouraged the belief that more of the same would eventually create life. Thus the Miller-Urey experiment was regarded as the first step on a road down which a chemical mixture would be inexorably conveyed by the input of energy and the passage of time. Today that assessment seems flawed. Amino acids are easy to make because they are thermodynamically favoured (like crystallization). In other words, their synthesis is a "downhill" process, needing no energy. Unfortunately the next step - linking together amino acids to form proteins - is an "uphill" process, requiring an input of energy. Admittedly, there was no lack of energy sources available to drive thermodynamically unfavourable processes in a pre-biotic setting (e.g. sunlight, geothermal energy). But just throwing energy willy-nilly at a random assemblage of amino acids is no more likely to produce a protein than a stick of dynamite exploded under a pile of bricks is likely to produce a house. In both cases the essential building blocks must be assembled in a highly specific and elaborate arrangement. In the case of DNA, chemistry determines the helical structure and explains the base-pair bonding, but chemistry is completely indifferent to the all-important sequence of base pairs - the organism's blueprint. Therefore I do not think that chemistry contains a "life principle". Belief that it does stems from the nineteenth century idea that life is some sort of magic matter, which can be cooked up from scratch by knowing the recipe, as if baking a cake (Davies 1998).

I should like to conjecture in this paper that quantum mechanics might provide de Duve's cosmic imperative. The founders of quantum mechanics certainly believed something like this. Fifty years ago quantum physicists, fresh from their success in explaining the atomic and subatomic nature of matter, were convinced they were on the verge of explaining life too. Erwin Schrödinger wrote in his famous book What is Life? that "we must be prepared to find a new kind of physical law operating in it" (Schrödinger 1944). Similar comments were made by Niels Bohr, Eugene Wigner and others.

Today, however, these early expectations have not been fulfilled. Obviously at some level life is quantum mechanical, but there is a tacit assumption that quantum physics yields only the "tool kit" needed for life. It explains the chemical bonds, and the sizes and shapes of molecules, but it is irrelevant to the serious business of life, which depends more on the "lego principle". This is the idea that molecules are simple blocks that fit together like lego bricks, and that life's magic has to do with the organizational arrangement of these blocks and not with quantum mechanical processes per se.

But maybe we are thinking about life in the wrong way. I do not propose to review the vexed issue of defining life, but there is one property that is found only in living organisms or their artifacts, and that is semantic information. Life's secret lies with its ability to process and replicate information that is 
meaningful in a certain molecular milieu (the cell) ${ }^{1}$. DNA is a genetic database that contains digital instructions in mathematical code. These instructions have to be interpreted by ribosomes and tRNA and translated into the assembly of proteins. Thus the living cell is more supercomputer than magic matter. I suggest that this crucial informational basis of life opens the door to a quantum mechanical "life principle".

It was actually Schrödinger himself who pointed out the informational basis of life when he described in his book that the genetic record would be imprinted on "an aperiodic crystal". This was a remarkable insight. Normal crystals don't fit the bill because they are devoid of information: a crystal's structure is fixed merely by specifying the inter-atomic spacing. In this respect, crystals provide an example of a state of matter that is written into the laws of physics. The geometrical symmetries of a crystal reflect the underlying symmetries in those laws. As a result, there is a principle of "crystalline determinism" in the laws of physics. But life's key information content requires a structure that is complex, not simple. This can be made precise by appealing to a branch of mathematics known as algorithmic information theory (Chaitin 1990), which seeks to quantify the degree of complexity in a sequence (which could be a basepair sequence of nucleotides) in terms of the information content of the shortest algorithm that can generate or simulate that sequence. According to this definition, information-rich sequences are also random sequences. The existence of any pattern or regularity, even if subtly hidden, implies a wasteful redundancy in information-carrying capacity. Since we expect life to have optimized its information storage and processing labour, we expect base-pair sequences (and hence amino acid sequences) to be random too (Yockey 1992).

However, to say that the base-pair sequences in DNA are random is not to say that they are arbitrary. The specific sequences of amino acids that make a functional protein, for example, form a tiny subset in the set of all random sequences (almost all sequences are random). The situation is comparable to the words in a novel. Other combinations of words make meaningful sequences too (other stories), but the overwhelming majority of random word sequences are gobbledygook. In the same way the overwhelming majority of base-pair sequences are biological gobbledygook.

When it comes to the origin of life, we are faced with the need to explain the origin of biological information, which means the existence of structures that simultaneously possess two seemingly conflicting properties: randomness and specificity. The DNA and amino acid sequences are random, but belong to a very special, specific subset of random sequences. Randomness is not hard to generate in physics: chaotic systems, for example, are intrinsically random. Nor is specificity hard to achieve: the laws of physics are regularities that determine, or specify, particular states of matter. It is the combination that is so baffling. The situation is analogous to pouring coffee beans from a jar to make a random pattern. That in itself would be relatively easy, as would be the case of creating

\footnotetext{
${ }^{1}$ Of course, all physical systems can be considered as information processors in a trivial sense. By specifying their initial state digitally as a computational input, the final state at some later time might be considered the output. But this information is not semantic; it has no meaning and cannot be considered to constitute a set of instructions, as is the case for a gene (Küppers 1985).
} 
a simple pattern, such as a uniform distribution or a periodic array. But if what was required was a specific random pattern we would have to work very hard (or long) to achieve it (Davies 1998).

Nature does have a mechanism for generating specific randomness: it is Darwinian evolution. Darwinism deploys just the combination of chance and law to create specified complexity. Thus a short, random, specific genome may evolve, over an immense number of steps, into a long, random, specific genome. Specific random information is added. But Darwinian evolution kicks in only after life has started, so we cannot appeal to it as an explanation for the origin of life. This raises the question of when life begins. What is the smallest replicating molecular system that can undergo the Darwinian imperatives of variation and selection? We do not know the answer to this, although some estimates suggest the system must already be very large and complex. Certainly the smallest autonomous living organism known today is exceedingly complex.

Nevertheless, some people have suggested that an explanation for biogenesis may be found in the assumption of "Darwinism all the way down". They conjecture that there is a set of molecules simple enough to form by chance in a plausible pre-biotic setting that would kick-start the Darwinian game. A long phase of molecular evolution would then lead to something like the RNA world (Cech 1986), from which life as we know it, based on nucleic acids and proteins, might emerge. The problem with this theory is that the RNA world is already rather complex, and would represent just one branch on a vast decision tree of chemical pathways leading from the hypothetical simple first replicator molecule. So the problem of the origin of life reduces to explaining how the pre-biotic state navigated its way through this decision tree and "discovered" something like life as we know it, given the stupendous number of alternative chemical pathways that lead nowhere biologically interesting.

It is at this point that quantum mechanics suggests a solution. Recent work in the field of quantum computation has established that quantum systems process information exponentially more efficiently than classical systems (Milburn 1998). The secret of the awesome processing power of quantum computation lies with the twin properties of superposition and entanglement. A quantum superposition effectively allows a particle to be in more than one configuration at the same time. Entanglement means that a collection of particles may be linked in exponentially more ways than by mere classical juxtaposition. For example, $\mathrm{n}$ particles may exist in $2^{n}$ quantum combinations, but only $n$ classical combinations. By attaching information to each configuration, even a small quantum system may evolve a vast amount of information simultaneously. In effect, a quantum computer employs massive parallelism care of Mother Nature.

For a system to process information quantum mechanically, quantum coherence has to be maintained. A quantum particle such as an electron or an atom is described by a wave, and the information is associated with the amplitude of the wave. Waves may be combined together coherently to process the information. If this is done, spectacular improvements in computational power are achieved. It must be stressed that this technology is in its infancy, but it does prompt us to wonder whether Nature might have spotted the opportunities of quantum information processing and exploited them. (If not, the quantum computer would be the first piece of human technology that Nature had not discovered!) And 
where would be more likely than in that great digital information processing system, the living cell?

Quantum information processing has been shown to be effective in a wide range of search problems. For example, Farhi \& Gutmann (1998) have established that there is an exponential improvement in the efficiency of searching a decision tree, which prompts the speculation that the "discovery" of the RNA world by a chemical mixture navigating a pathway through a chemical decision tree might be considerably sped up if assisted by a quantum search strategy, at least in part.

More direct, albeit circumstantial, evidence that quantum information processing might have been harnessed by life comes from the work of Patel (2001), who applies Grover's algorithm (Grover 1999) to the genetic code. Grover's algorithm concerns the use of a quantum computer to search an unsorted database of $N$ objects of $Q$ varieties, which affords a $\sqrt{N}$ improvement in search effort. The exact solution of the algorithm is

$$
(2 Q+1) \sin ^{-1}\left(\frac{1}{\sqrt{N}}\right)=\frac{\pi}{2}
$$

Patel points out that for $Q=1, N=4$. This is a unique integer solution. For $Q=3, N=20.2$. It is indeed remarkable that Grover's algorithm throws up the numbers 4, 3 and 20 (or 21), which Patel associates with the number of nucleotides, the triplet code and the number of amino acids that life uses.

So far this is just numerology, but Patel suggests that DNA forms base pairs by actually performing a quantum computation. To this end, he points out that quantum superpositions of nucleotides will be presented to any given DNA base, and the 'right' one searched for. In doing this search quantum mechanically, using four nucleotides and a triplet base, DNA would be able to halve the search time. Thus Patel offers a quantum mechanical explanation for why life employs four nucleotides rather than two to encode information.

There have been other suggestions that living systems process information quantum mechanically. McFadden (2000) has developed a theory of evolution that appeals to quantum tunnelling in base pair formation. Nanopoulos and his co-workers have proposed that the protein tubulin can form an array of quantum binary switches that could function as a quantum cellular automaton (Mershin et al. 2000). Penrose and Hameroff believe that some information in the brain is processed at the sub-neuronal level in microtubules (Penrose 1994; Hameroff 1998). These claims have meet met with a degree of scepticism (see for example, Tegmark 2000).

A central objection to quantum mechanical explanations of biological processes is the phenomenon of decoherence (Zurek 1991). As explained, information may be processed reliably only if the quantum waves involved remain coherent, i.e. their delicate phase relationships are undisturbed. Many quantum systems decohere extremely rapidly by coupling to noisy environments. This has the effect of scrambling the crucial phase relationships and driving up the entropy. The upshot is that, as far as the information processing aspects are concerned, the behaviour of the system effectively reverts to being classical. For example, a simple calculation (Tegmark 2000) indicates that activity in brain microtubules will decohere on a timescale of less than $10^{-13} \mathrm{~s}$. Unless coherence 
can be maintained for biochemically relevant time scales, quantum information processing is unlikely to play a significant role in biology.

Two possible ways around this obstacle present themselves. The first is screening. Patel points out (Patel 2001) that during DNA replication the active region is fully enveloped by the replicase protein, which will serve to shield the nucleotides from external decohering disturbances. Moreover, such enzymes may act to reduce quantum mechanical barriers, and thus greatly amplify quantum coherence effects. Patel conjectures that some enzymes might actually be selected for according to their quantum mechanical prowess in this regard.

The second way of combating decoherence is to turn a sin into a virtue and actually exploit coupling to a noisy environment. Various quantum phenomena such as the watch-dog, or Zeno, effect (Itano et al. 1990) are known to effectively freeze the status quo of a quantum system strongly interacting with an environment. Similar strong coupling can produce "decoherent-free subspaces" - regions of the state space that are quiescent and immune from external disturbances.

An example of such a phenomenon is provided by the calculations of Bell, Sawyer \& Volkas (2002). They consider the case of a double potential well. A quantum particle in one well may tunnel into the other, and in the ground state it can be considered as oscillating back and forth between the two wells with a certain frequency. Excited states can occur in the wells too, and they also oscillate, but with different frequencies. If the system is started out in a superposition of states, the phases will soon get out of kilter due to these differing oscillation frequencies. But now suppose the system is coupled to an external heat bath (source of noise). For low coupling strengths the phases get scrambled even faster, and the entropy of the system rises as it loses information to the environment. This is the bugbear of environmental decoherence robbing the system of its computational fidelity. However, when the coupling to the heat source becomes large, an amazing thing happens. The quantum waves associated with the various states all start to oscillate in phase (with a period determined by the average frequency of the oscillators). In the limit of infinitely strong coupling, perfect coherence is re-established! If we consider a pair of entangled particles strongly coupled to the heat source, entanglement is preserved in the face of the external noise.

Could it be that biological systems are able to exploit these coherencefreezing properties of certain noisy quantum systems, at least long enough to carry out some information processing tasks? Whilst it would be rash to suppose that a living cell might actually be a functioning quantum computer, some quantum-assisted information processing may take place. Should this be the case, it may pay for physicists working on the engineering aspects of quantum computation to see whether life may teach them a few tricks.

Regarding the problem of life's origin, the subject of this paper, I venture the following prediction. The coming decades will see the merging of nanotechnology, biotechnology and quantum technology. The central theme around which all these disciplines revolve is information processing. A century ago scientists dreamed of using chemistry to make life de novo. I have argued that chemistry may be the medium of life's origin, but one must not confuse the medium with the message, i.e. the information content. I do not believe we will cook up life 
in a test tube from some chemical recipe. I think that life will eventually be created artificially, but as a by-product of quantum information processing and nanotechnology, not of organic chemistry.

Acknowledgments. I should like to thank Derek Abbott, Jim Al-Khalili, Nicole Bell and Gerard Milburn for helpful discussions on this topic.

\section{References}

Bell, N. F., Sawyer, R. F., \& Volkas, R. R. 2002, Phys.Rev.A, Entanglement and quantal coherence: a study of two limiting cases of rapid systembath interactions

Cech, T. 1986, Scientific American 255, No. 5, RNA as an enzyme, 64

Chaitin, G. 1990, Information, Randomness \& Incompleteness: Papers on Algorithmic Information Theory, second edition (Singapore: World Scientific Press)

Davies, P. 1998, The Fifth Miracle: The Search for the Origin of Life (London: Penguin)

De Duve, C. 1995, Vital Dust, (New York: Basic Books)

Farhi, E. \& Gutmann, S. 1998, Phys.Rev.A58, Quantum computation and decision trees, 915

Grover, L. 1999, The Sciences, July/August edition, Quantum computing, 24

Hameroff, S. R. 1998, Phil. Trans. Royal Soc. (London) A356, Quantum computation in brain microtubules? The Penrose-Hameroff "Orch OR" model of consciousness, 1869

Itano, W. M. Heinzen, D. J., Bollinger, J. J. \& Weinland, D. J. 1990, Phys.Rev.A41, Quantum Zeno effect, 2295

Küppers, B. O. 1985, Molecular Theory of Evolution, (Berlin: Springer-Verlag)

McFadden, J. 2000, Quantum Evolution, (London: HarperCollins)

Mershin, A., Nanopoulos, D. V. \& Skoulakis, E. M. C. 2000, LANL preprint quant-ph, Quantum brain?, 0007088n

Milburn, G. 1998, The Feynman Processor, (Reading, Mass.:Perseus Books)

Miller, S. L. 1953, Science 117, 528

Patel, A. 2001, Pramana 56, 367

Penrose, R. 1994, Shadows of the Mind, (Oxford: Oxford University Press)

Schrödinger, E. 1944, What is Life?, (Cambridge: Cambridge University Press)

Shapiro, R. 1986, Origins: A Skeptic's Guide to the Creation of Life on Earth (New York: Summit Books)

Tegmark, M. 2000, Phys. Rev. E 61, 4194

Yockey, H. 1992, Information Theory and Molecular Biology, (Cambridge: Cambridge University Press)

Zurek, W. H. 1991, Physics Today, 44, 36 


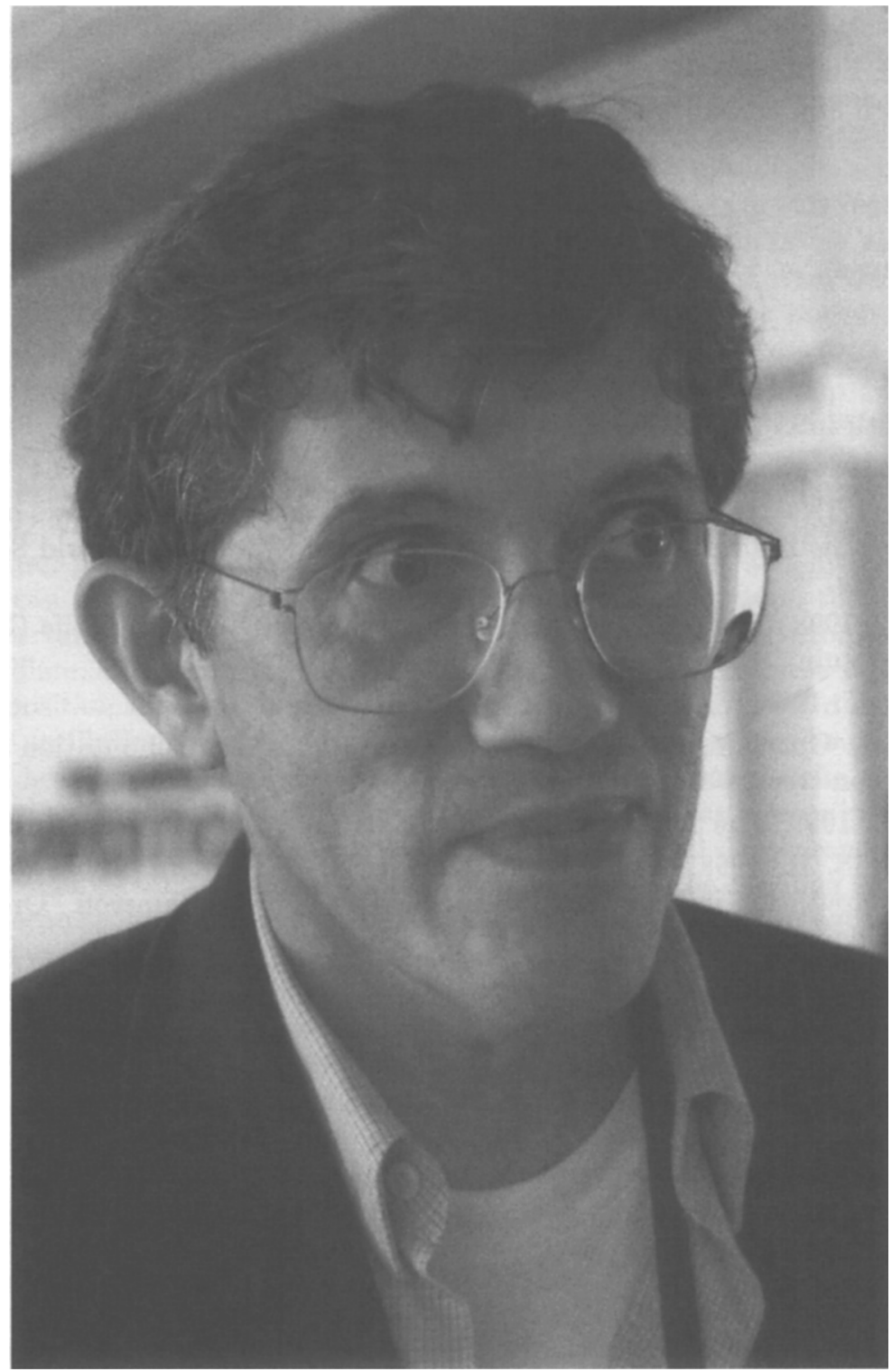

Antonio Lazcano (photo: Seth Shostak) 